http://jmscr.igmpublication.org/home/ ISSN (e)-2347-176x ISSN (p) 2455-0450 crossref DOI: https://dx.doi.org/10.18535/jmscr/v9i1.53

\title{
Budesonide Combination A Better Clinical Approach in Comparison to Cyclosporine in Treatment of Autoimmune Hepatitis
}

\author{
Authors \\ Rasmirekha Behera ${ }^{1}$, Sushant Sethi ${ }^{2}$ \\ ${ }^{1}$ Dept of Pharmacology I.M.S \& SUM Hospital Bhubaneswar, India \\ ${ }^{2}$ Dept of Gastroenterology Apollo Hospital Bhubaneswar, India
}

\begin{abstract}
Autoimmune hepatitis $(A I H)$ is an uncommon though serious and potentially life-threatening disease which requires prompt recognition and treatment. In cases unresponsive to conventional treatment, achieving disease remission can be difficult. Keeping this in background in this study a comparision has been made between Budesonide combination with that of immunosuppressant i.e cyclosporine in treatment of Autoimmune Hepatitis.

Methods: 30 AIH patients were included in this study. All patients were divided into two groups i.e group $A$ and B.15 patients Group A were treated with Budesonide $9 \mathrm{mg} / \mathrm{kg} /$ day with Azathioprine $2 \mathrm{mg} / \mathrm{kg} /$ day and 15 patients Group B were treated with Cyclosporine $3 \mathrm{mg} / \mathrm{kg} /$ day. All the drugs were administered for 6 months.

Result: All the patients with Budesonide and Azathioprine therapy were found to Antinuclear antibody negative after 6 months and more than 10 patients of Group A were found to have decrease in level of AST and ALT after 6 months. In patients with Cyclosporine therapy only 9 patients were found to be ANA negative after 6 months. Less than 10 patients were found to have decrease in levels of AST and ALT after 6 months in group $B$.

Conclusion: Group A patients demonstrated a good biochemical response after 6 months of therapy in comparision to Group B patients. From this it is concluded that patients with Budesonide and Azathioprine therapy shows a significant improvement in AIH after 6 months in comparision to Cyclosporine therapy.

Keywords: Autoimmune Hepatitis, Budesonide, Azathioprine, Cyclosporine, Alanine aminotransferrase, Aspartate aminotransferase, Antinuclear Antibody, Immunosupressant.
\end{abstract}

\section{Introduction}

Autoimmune hepatitis (AIH) is a complex immune mediated liver disease that is diagnosed histologically by interface hepatitis and high serum levels of alanine aminotransferase (ALT), aspartate aminotransferse (AST), and immunoglobin $\mathrm{G}$ (IgG) and presence of autoantibodies. ${ }^{(1)}$ AIH can be asymptomatic or present in various forms from subclinical disease to acute liver failure and end-stage liver disease. ${ }^{(2)}$ AIH is divided in Type 1 and Type 2, the latter being rare in adults and representing 30\% of juvenile AIH. The distinction is made serologically: type $1 \mathrm{AIH}$ is positive for antinuclear antibodies (ANA), and/or anti-smooth muscle antibodies (SMA), while type $2 \mathrm{AIH}$ is 
positive for anti-liver kidney microsomal antibodies type 1(anti-LKM 1) and/or anti-liver cytosol type 1 (anti-LC1) ${ }^{(3)}$ The exact mechanisms for the immune tolerance break-down in AIH have not been described yet, but there is growing evidence that a genetic predisposition, molecular mimicry, and an imbalance between effector and regulatory immunity are key pathologic components for disease development. Several lines of evidence support the central role of impaired $\mathrm{T}$ cell number and function. ${ }^{(1)}$ Further putative triggers (e.g viruses) for AIH have also been linked to the hypothesis of molecular mimicry and cross-reactivity between foreign epitopes and hepatic antigens ${ }^{(4)}$. This includes hepatitis A virus(HAV) ${ }^{(5)}$ hepatitis $\mathrm{C}$ virus $(\mathrm{HCV})^{(6)}$, hepatitis $\mathrm{E}$ virus $(\mathrm{HEV})^{(7)}$ measles $^{(8)}$ Epstein-Barr virus $(\mathrm{EBV})^{(9)}$ and herpes simplex virus. $^{(10)}$ Several drugs (e.g minocycline, nitrofurantoin, melatonin, diclofenac, statins and orinidazole which may be involved in precipitating $\mathrm{AIH}$. It is important to clarify that drug-induced $\mathrm{AIH}$ is completely different entity from drug-induced liver injury (DILI); however, overlap syndromes have been described in up to 9\% of cases in which AIH and DILI are indistinguishable from each other. ${ }^{(11)}$ The diagnosis of AIH is based on the presence of specific autoantibodies, immunoglobulin levels and histology as well as the absence of acute viral serology. ${ }^{(12,13)}$ Anti-SLA (anti-soluble liver antigen) is highly specific for the diagnosis of AIH. ${ }^{(14,15)}$ The classical histological hallmark of $\mathrm{AIH}$ is interface hepatitis characterized by inflammation and erosion at the junction of the hepatic parenchyma with the portal tracts. Centrilobular lesions and necrosis are present when the disease is severe and progressive. Acute cases may appear histologically indistinct to drug induced liver injury. ${ }^{(16)}$ Fibrosis and cirrhosis may already be evident in subacute disease. ${ }^{(12)}$

Absolute indications for treatment are a serum AST greater than 10 times the upper limit of normal or an AST greater than 5 times the upper limit of normal in conjuction with a serum globulin level greater than 2 times the upper limit of normal. Bridging or multilobular necrosis at presentation is an absolute indication for treatment given the risk of progression to cirrhosis. ${ }^{(17)}$ Furthermore incapacitating systemic symptoms such as fatigue and arthralgia are also considered absolute indications for treatment. ${ }^{(17)}$ Diseases that can resemble autoimmune hepatitis must also be excluded by appropriate tests and these include virus-related, drug-induced, alcoholic, hereditary (Wilson disease, hereditary hemochromatosis), meta-bolic (nonalcoholic fatty liver disease (NAFLD), and immune-mediated cholestatic diseases (PBC and PSC). ${ }^{(18)}$ The aim of treatment is disease remission, which is reached if the following criteria are met:(1)absence of clinical symptoms; (2 )normal transaminase levels; and (3) normal IgG levels.In children /adolescents, negative or very low-titre autoantibodies $(<1: 20$ for ANA/SMA; $<1: 10$ for anti-LKM1) are an additional criterion of remission.(19)Formalized diagnostic criteria ensure the application of a standardized diagnostic algorithm, ${ }^{(20)}$ and diagnostic scoring system provide an evaluation template that can support the diagnosis in difficult cases. $^{(20,21,22)}$ Successful treatment aims at reducing inflammation and preventing progressive fibrosis while minimizing side effects associated with therapy ${ }^{(23)}$ Standard treatment approaches have remained static for decades, since Prednisolone with or without azathioprine (AZA) was first introduced in the 1950s - 1960s. ${ }^{(24)}$ Although definitions of therapeutic endpoints were heterogenous across the reviewed studies, most defined treatment response as resolution of clinical symptoms and normalization of transaminase and immunoglobulin $\mathrm{G}$ ( $\mathrm{IgG}$ ) levels within 6 months after initiation of therapy. $(25,26,27,28)$ Currently, initial standard therapy consists of corticosteroids (prednisone or prednisolone) with or without AZA. ${ }^{(23,29)}$ Most patients respond very well to standard therapy If appropriately managed, and alternative treatment modalities are needed only for the minority whom cannot tolerate or do not respond 
to usual approaches.Overall,10-15\% of patients on standard therapy discontinue due to intolerable side effects. ${ }^{(30)}$ and up to $18 \%$ of those who present with jaundice fail initial treatment. ${ }^{(31)}$ Budesonide is a synthetic glucocorticoid with a more than $90 \%$ hepatic first pass effect. It is associated with low corticosteroidal bioavailability and low steroid specific side effects. ${ }^{(32)}$ Bone marrow suppression is the major side effect of azathioprine, whereas steroid specific side effects, such as moon face, acne, buffalo hump, hirsutism, striae, diabetes, and glaucoma, often occur in patients treated with prednisolone. ${ }^{(33)}$ The frequency of side effects was low Budesonide is an appropriate induction option in treatment-naïve patients without advanced fibrosis, in those with steroid-related side effects, and in those at risk of adverse effects for steroids including those with metabolic bone disease and brittle diabetes. The presence of obesity, hypertension, or osteopenia that might be worsened by prednisone treatment also support consideration of the budesonide regimen. ${ }^{(34)} \mathrm{A}$ simplified diagnostic scoring system has been developed to ease clinical application. ${ }^{(21)}$ It evaluates four clinical categories and renders nine possible grades. ${ }^{(21)}$ The original revised scoring system has greater sensitivity for autoimmune hepatitis $(100 \%$ vs $95 \%){ }^{(22)}$ whereas the simplified scoring system has superior specificity (90\% vs $73 \%$ ) and accuracy(92\% vs $82 \%$ ), using clinical judgment as the gold standard. ${ }^{(22)}$ Oral budesonide is an alternative to prednisolone and lessens systemic steroid side effects. It can be given at doses of $3 \mathrm{mg}$ twice or thrice daily in combination with azathioprine. ${ }^{(35)}$ Budesonide in combination with azathioprine has emerged as an alternative frontline treatment for autoimmune hepatitis. ${ }^{(34)}$ Cyclosporine has been administered in doses of 2 to $5 \mathrm{mg}$ body weight with dose adjustments to achieve through levels of 100 to $300 \mathrm{ng} / \mathrm{ml} .^{(34,36,37)}$ Cyclosporine A belong to the group of calcineurin inhibitors (CNI) which find widespread application as immunosuppressive drugs by inhibiting $\mathrm{T}$ cell activation and IL-2 production. ${ }^{(38)}$ In this study the treatment outcome is compared between Budesonide with azathioprine and Cyclosporine in patients of autoimmune hepatitis.

\section{Material and Methods}

The study was conducted in the department of Gastroenterology Apollo Hospital Bhubaneswar for 6 months from October 2019 to March 2020.

\section{Inclusion Criteria}

1. Patients are selected between 18 to $60 \mathrm{yrs}$ diagnosed with Autoimmune Hepatitis with Obesity, Hypertension and Diabetes.

2. Disease refractory to steroids and azathioprine.

3. Patients with bone marrow suppression $\&$ opportunistic infection due to Azathioprine

4. Patients with prednisolone dependence and steroid side effects.

\section{Exclusion Criteria}

1. Patients above $60 \mathrm{yrs}$ and below $18 \mathrm{yrs}$ of age.

2. Patients with cirrhosis.

Patients were divided into two groups i.e Group: A and Group: B. In each group 15 patients were included. Group A patients were given Budesonide 9mg/day with Azathioprine with a dose of $2 \mathrm{mg} / \mathrm{kg} / \mathrm{day}$. Group B patients were given Cyclosporine $3 \mathrm{mg} / \mathrm{kg} /$ day. The drugs were given for 6 months to both the groups. Patients diagnosed with AIH with Obesity, Hypertension, Diabetes and with steroid dependence are considered for Group A and given Budesonide and Azathioprine combination. Patients diagnosed with AIH but refractory to steroids and Azathioprine therapy and showing bone marrow suppression, opportunistic infection are categorized as Group B and considered for cyclosporine therapy.

The diagnostic criteria of the IAIHG require the presence of compatible laboratory serum aspartate (AST) and alanine aminotransferase (ALT) abnormalities, hypergammaglobulinemia and increased serum IgG level, serological (ANA, SMA or anti-LKM1 positivity and histological 
findings (interface hepatitis with or without plasma cell infiltration. ${ }^{(20)}$

Statistical Analysis: Statistical Analysis was done by applying paired t-test. As there are 30 samples degree of freedom is 29.P value found to be less than 0.05 and the difference observed is significant.

\section{Result}

Result were compared with values of AST, ALT and ANA. Evaluation of all the parameters were done at 0 month, 1 month, 3month and 6month intervals. Values of ALT \& AST represented in $\mathrm{U} / \mathrm{L}$

Table:1 Budesonide with Azathioprine Therapy (Group: A 15 patients)

\begin{tabular}{|c|c|c|c|c|}
\hline Parameters & 0 month & 1 month & 3 month & 6 month \\
\hline AST & 650 & 550 & 300 & 60 \\
\hline ALT & 120 & 100 & 90 & 45 \\
\hline ANA & +++ & ++ & - & - \\
\hline
\end{tabular}

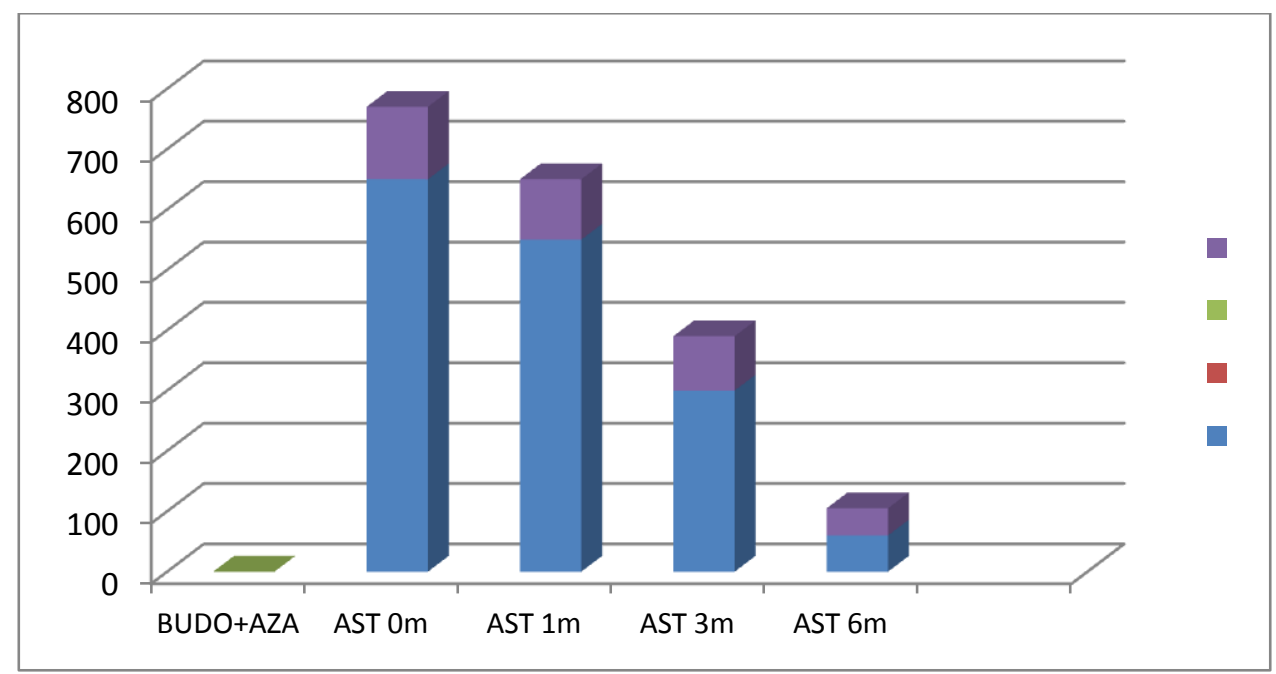

Graph-1 shows enzyme level after1,3 \& 6 months of Budesonide and Azathioprine therapy

Table: 2 Cyclosporin Therapy (Group:B 15 patients)

\begin{tabular}{|c|c|c|c|c|}
\hline Parameters & 0 month & 1 month & 3 month & 6 month \\
\hline AST & 670 & 650 & 500 & 350 \\
\hline ALT & 140 & 130 & 110 & 90 \\
\hline ANA & +++ & ++ & ++ & + \\
\hline
\end{tabular}

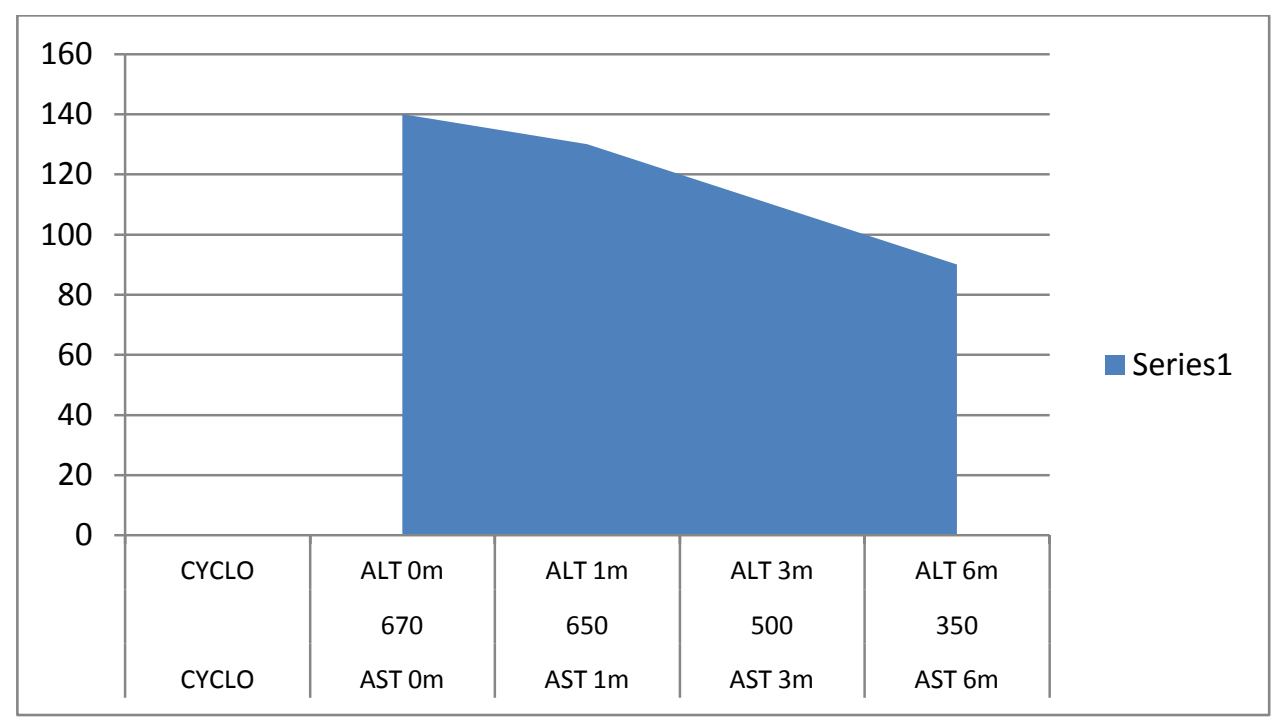

Graph: 2- shows enzyme levels after 1,3 and 6 months of Cyclosporine therapy 


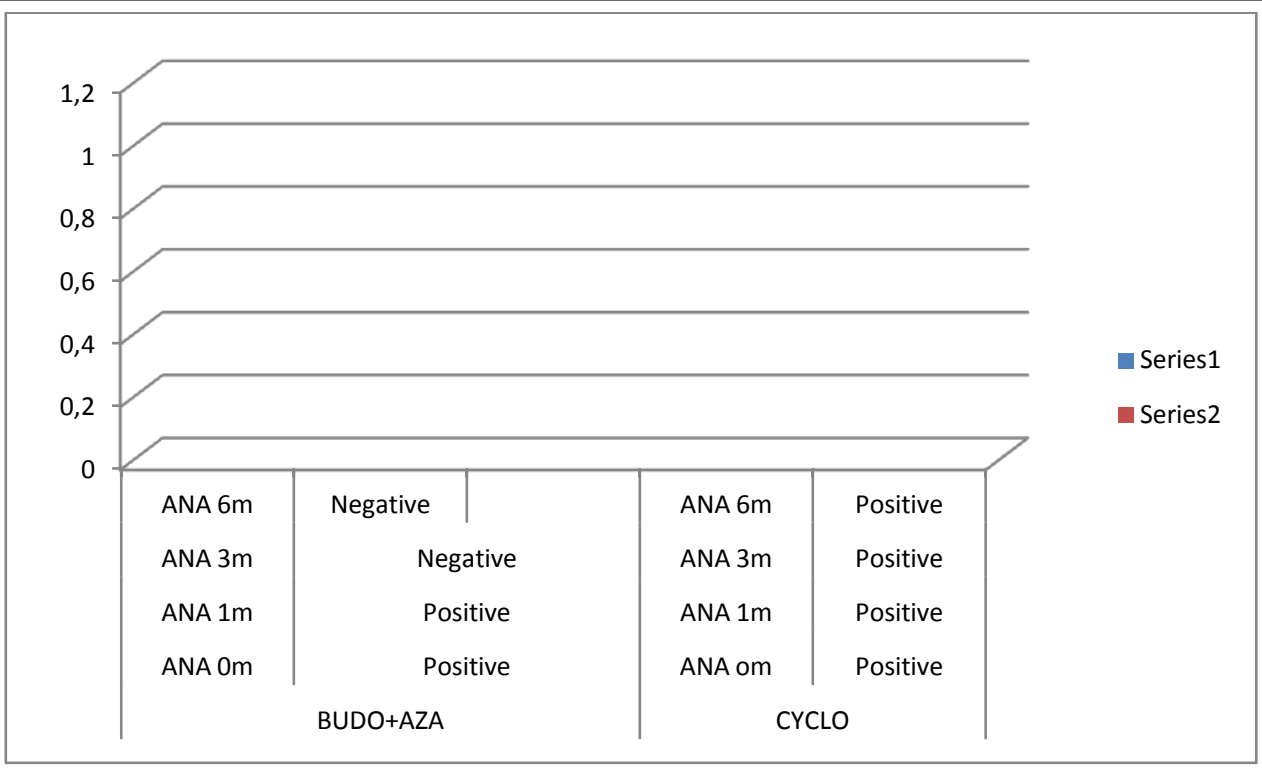

Graph: 3 shows the comparision of ANA responses after Budosonide with Azathioprine therapy and Cyclosporine therapy

Table 3 Group: A Budesonide with Azathioprine (15 patients) Response after 1,3,6 months represented in numbers

\begin{tabular}{|l|c|c|c|}
\hline \multicolumn{4}{|c|}{ Budesonide and Azathioprine Therapy } \\
\hline Parameters & 1 month & 3 month & 6 month \\
\hline$\downarrow$ in AST & 6 & 8 & 13 \\
\hline$\downarrow$ in ALT & 7 & 9 & 12 \\
\hline ANA- & 5 & 13 & 15 \\
\hline
\end{tabular}

Table 4 Group: B Cyclosporin Therapy (15 patients) Response after 1,3,6 months represented in numbers

\begin{tabular}{|l|c|c|c|}
\hline \multicolumn{4}{|c|}{ Cyclosporine Therapy } \\
\hline & 1 month & 3 month & 6 month \\
\hline$\downarrow$ in AST & 5 & 6 & 7 \\
\hline$\downarrow$ in ALT & 6 & 7 & 8 \\
\hline ANA- & 4 & 6 & 9 \\
\hline
\end{tabular}

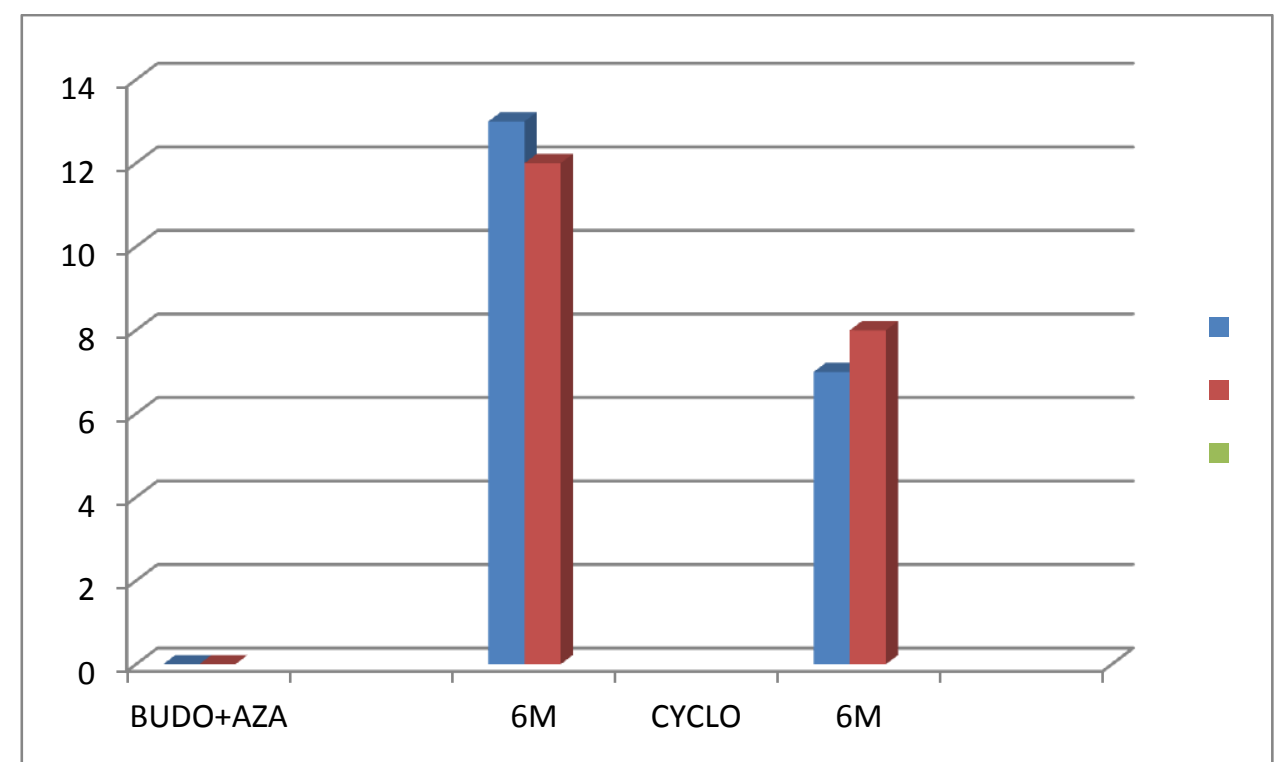

Graph: 4 shows the Comparision of responses between Budesonide with Azathioprine therapy and Cyclosporine Therapy (Represented in no) 
It is observed from the above table 3 and table 4 that all the patients with Budesonide and Azathioprine therapy were found to Antinuclear antibody negative after 6 months of drug administration. More than 10 patients were found to have decrease in level of AST and ALT after 6 months of drug administration. In patients with Cyclosporine therapy only 9 patients were found to be ANA negative after 6 months of drug administration \& less than 10 patients were found to have decrease in levels of AST and ALT. Graph 1 \& 2 shows that there is decrease in enzyme levels after administration of drugs in both Group A and Group B. But the decrease in enzyme level is more in Group A in comparision to Group B. It signifies better response of disease activity in Budesonide with Azathioprine administration in comparision to Cyclosporine therapy. Again from the graph 4 it is observed that no of patients showing improvement in all parameters after 6 months of drug administration is more in Group A. Graph 3 shows all the patients in Group A are ANA negative after 6 months of drug administration.

\section{Discussion}

Budesonide is a synthetic corticosteroid with topical anti-inflammatory properties and less steroid-specific side effects due to high first pass hepatic metabolism. Budesonide is a less desirable option in patients with cirrhosis due to impaired hepatic metabolism and increased systemic bioavailability, as well as the potential for increased risk of portal vein thrombosis. ${ }^{(39,40)}$ Most patients respond very well to standard therapy if appropriately managed and alternative treatment modalities are needed only for the minority whom cannot tolerate or do not respond to usual approaches.Overall,10\%-15\% of patients on standard therapy discontinue due to intolerable side effects and up to $18 \%$ of those who present with jaundice fail initial treatment. ${ }^{(41,42)}$ Cyclosporine is calcineurin inhibitor highly effective for prevention of graft rejection reaction. Cyclosporine reported to be effective in variable doses and duration of treatment in patients of AIH not responding to Azathioprine and Prednisolone. ${ }^{(43)}$

\section{Conclusion}

The present study shows improvement with Budesonide and Azathioprine therapy in all parameters after 6months of drug administration. Group A patients demonstrated a good biochemical response after 6 months of therapy in comparision to Group B patients.Also the Antinuclear antibody were found to be negative in all patients with Budesonide and Azathioprine therapy after 6 months. From this it is concluded that patients with Budesonide and Azathioprine therapy shows a significant improvement in $\mathrm{AIH}$ after 6 months in comparision to Cyclosporin therapy.

\section{References}

1. A. Floreani P, Restrepo-Jimenez, M.F. Secchi et al," Etiopathogenesis of autoimmune hepatitis", Journal of Autoimmunity,vol 95,pp.133-143,2018.

2. A.J. Czaja,'Diagnosis and management of autoimmune hepatitis: current status and future directions," Gut Liver, vol.10, no.2,pp.177-203,2016.

3. Vergani D, Mieli-Vergani G. Pharmacological management of autoimmune hepatitis. Expert Opin Pharmacother 2011;12:607-613.

4. M.A. Heneghan, A.D.Yeoman, S.Verma, A.D. Smith and M.S. Longhi," Autoimmune hepatitis," Lancet, vol.382, no.9902, pp.1433-1444,2013.

5. S.Vento,T.Garofano, G.Di Perri, L.Dolci, E. Concia, and D. Bassetti," Identification of hepatitis A virus as a trigger for autoimmune chronic hepatitis type 1 in susceptible individuals," The Lancet, vol .337,no.8751,pp.1183-1187,1991.

6. S.Vento, F.Cainelli, C. Renzini, E.Concia," Autoimmune hepatitis type 2 induced by $\mathrm{HCV}$ and persisting after viral 
clearance,"Lancet,vol.350,no.9087,pp.129 8-1299,1997.

7. P.Le Cann, M.J.Tong, J.Werneke, and P. Coursaget," Detection of antibodies to hepatitis $\mathrm{E}$ virus in patients with autoimmune chronic active hepatitis and primary billiary cirrhosis," Scandinavian Journal of Gastroenterology, vol.32,no.4 pp.387-389, 1997.

8. S.Vento,L.F.Cainelli,T.Ferraro, and

E.Concia," Autoimmune hepatitis type 1 after measles," The American Journal of Gastroenterology, vol. 91,no.12,pp.2618$2620,1996$.

9. S.Vento, L.Guella, F.Mirandola, F.Cainelli, G. Di Perri, M.Solbiati et.al.," Epstein-Barr virus as a trigger for autoimmune hepatitis in susceptible individuals," Lancet, vol.346, no.8975, pp.608-609, 1995.

10. M.P.Manns,"'Viruses and autoimmune liver disease,'Inter-virology, vol.35,no.14,pp. 108-115,1993.

11. A. Licata, M.Maida, D.Cabiba et al.,"Clinical features and outcomes of patients with drug-induced autoimmune hepatitis: a retrospective cohort study," Digestive and liver Disease, vol.46, no.12, pp.1116-1120,2014.

12. European Association for study of the Liver. EASL clinical practice guidelines: Autoimmune hepatitis.J Hepatol 2015;63:971-1004.

13. Gleeson D, Heneghan MA. British Society of Gastroenterology (BSG) guidelines for management of autoimmune hepatitis.Gut 2011;60:1611-1629.

14. Bogdanos DP, Mieli-Vergani G, Vergani $\mathrm{D}$, Autoantibodies and their antigens in autoimmune hepatitis. Semin Liver Dis 2009;29:241-253.

15. Liberal R, Mieli-Vergani G,Vergani D. Clinical significance of autoantibodies in autoimmune hepatitis.J Autoimmune 2013;46:17-24.
16. Washington MK. Autoimmune liver disease: overlap and outliers. Mod Pathol 2007;20(Suppl 1):515-530.

17. Manns MP, Czaja AJ, Gorham JD, Krawitt EL et al. Diagnosis and management of Autoimmune hepatitis. Hepatology 2010;51:2193-2213.

18. Alvarez F, Berg PA, Bianchi FB, et al. International Autoimmune Hepatitis Group Report: review of criteria for diagnosis of autoimmune hepatitis. J Hepatol 1999;31:929-938.)

19. Vergani D, Mackay IR, Mieli-Vergani G. The Autoimmune Disease(Fifth Edition). Boston Academic Press;2014.p 889-907.

20. Alvarez F, Berg PA, Bianchi FB et al.I nternational Autoimmune Hepatitis Group Report: review of criteria for diagnosis of autoimmune hepatitis. J Hepatol 1999;31:929-938.

21. Hennes EM, Zeniya M, Czaja AJ, et al. Simplified criteria for the diagnosis of autoimmune hepatitis. Hepatology 2008;48:169-176.

22. Czaja AJ. Performance parameters of the diagnostic Scoring systems for autoimmune hepatitis. Hepatology 2008;48:1540-1548.

23. Manns MP, Czaja AJ, Gorham JD,et al. Diagnosis and management of autoimmune

hepatitis.Hepatology.2010;51:2193-2213.

24. Mackay IR. Historical reflections on autoimmune hepatitis. World J Gastroenterol.2008;14:3292-3300.

25. Peiseler M, Liebscher T, Sebode M, et al, Efficacy and limitations of budesonide as a second-line treatment for patients with autoimmune hepatitis. Clin Gastroenterol Hepatol.2018;16(260-267).

26. Zachou K, Gatselis NK. Arvaniti P.et al.A real-world study focused on the long-term efficacy of mycophenolate mofetil as firstline treatment of autoimmune hepatitis. 
Aliment Pharmacol Ther. 2016;43:10351047.

27. Efe C, Hagstrom H, Ytting $H$,et al. Efficacy and safety of mycophenolate mofetil and tacrolimus as second-line therapy for patients with autoimmune hepatitis. Clin Gastroenterol Hepatol. 2017;15(1950-1956).

28. Than NN, Wiegard C, Weiler-Normann C, et al. Long-term follow-up of patients with difficult to treat type 1 autoimmune hepatitis on Tacrolimus therapy.ScandJ Gastroenterol.2016;51:329-336.

29. European Association for the study of the liver. EASL clinical practice guidelines: autoimmune hepatitis J Hepatol.2015;63:971-1004.

30. Czaja A J.Safety issues in the management of autoimmune hepatitis. Expert Opin Drug Saf.2008:7:319-333.

31. Yeoman AD,Westbrook RH,Zen Y,et al.Early predictors of corticosteroid treatment failure in icteric presentations of autoimmune hepatitis.Hepatology.2011;53:926-934.

32. Danielsson A,Prytz H. Oral budesonide for treatment of autoimmune chronic active hepatitis. Aliment Pharmacol Ther 1994;8:585-590.

33. Manns MP, Czaja AJ, Gorham JD, et al.Diagnosis and management of autoimmune hepatitis. Hepatology 2010;51:2193-2213.

34. Czaja AJ. Current and prospective pharmacotherapy for autoimmune hepatitis. Expert Opin Pharmacother 2014;15:1715-1736.

35. Manns MP,Woynarowski M, Kreisel W, Lurie Y, Rust C, Zuckerman E, et al. Budesonide induces remission more effectively than prednisone in a controlled trial of patients with autoimmune hepatitis. Gastroenterology 2010;139:1198-1206.
36. Malekzadeh R, Nasseri-Moghaddam S,Kaviani MJ, Taheri H, Kamalian N, Sotoudeh M. Cyclosporin A is a promising alternative to corticosteroids in autoimmune hepatitis.Dig Dis Sci 2001;46:1321-1327.

37. Czaja AJ. Drug choices in autoimmune hepatitis. Part B:nonsteroids. Expert Rev Gastroenterol Hepatol 2012;6:617-635.

38. A Furukawa, S.A. Wisel, and Q Tang," Impact of immunomodulatory drugs on regulatory T cell," Transplantation, vol.100.no.11, pp.2288-2300,2016.

39. Geier A, Gartung C, Dietrich CG, Wasmuth HE, Reinartz P, Matern S. Side effects of budesonide in liver cirrhosis due to chronic autoimmune hepatitis: influence of hepatic metabolism versus portosystemic shunts on a patient complicated with HCC.World J Gastroenterol.2003;9:2681-2685.

40. Hempfling W, Grunhage F, Dilger K, Reichel C, Beuers U, Sauerbruch T. Pharmacokinetics and Pharmacodynamic action of budesonide in early and late stage primary biliary cirrhosis .Hepatology.2003;38;196-202.

41. Czaja AJ. Safety issues in the management of autoimmune hepatitis. Expert Opin Drug Saf.2008;319-333.

42. .Yeoman AD, Westbrook RH, Zen Y, et al. Early predictors of corticosteroid treatment failure in icteric presentations of autoimmune hepatitis.Hepatology.2011;53:926-934.

43. Alvarez F, Ciocca M, Canero-Velasco C, Ramonet $\mathrm{M}$ et al. Short-term cyclosporine induces a remission of autoimmune hepatitis in children. $\mathbf{J}$ Hepatol 1999;30:222-227. 